PAPER

\title{
Fair, just and compassionate: A pilot for making allocation decisions for patients requesting experimental drugs outside of clinical trials
}

\author{
Arthur L Caplan, ${ }^{1}$ J Russell Teagarden, ${ }^{2}$ Lisa Kearns, ${ }^{1}$ Alison S Bateman-House, ${ }^{1}$ \\ Edith Mitchell, ${ }^{3}$ Thalia Arawi, ${ }^{4}$ Ross Upshur, ${ }^{5,6}$ llina Singh, ${ }^{7}$ Joanna Rozynska, ${ }^{8}$ \\ Valerie Cwik, ${ }^{9}$ Sharon L Gardner ${ }^{10}$
}

For numbered affiliations see end of article.

\section{Correspondence to}

Dr Arthur L Caplan, Division of Medical Ethics, NYU School of Medicine, New York City, NY 10016, USA ;

Arthur.Caplan@nyumc.org

Received 15 March 2018 Revised 6 June 2018 Accepted 16 June 2018 Published Online First 7 July 2018

\section{Linked}

- http://dx.doi.org/10.1136/ medethics-2016-103474

Check for updates

(C) Author(s) (or their employer(s)) 2018. No commercial re-use. See rights and permissions. Published by BMJ.

To cite: Caplan $A L$, Teagarden JR, Kearns L, et al. $J$ Med Ethics

2018;44:761-767.

\section{ABSTRACT}

Patients have received experimental pharmaceuticals outside of clinical trials for decades. There are no industry-wide best practices, and many companies that have granted compassionate use, or 'preapproval', access to their investigational products have done so without fanfare and without divulging the process or grounds on which decisions were made. The number of compassionate use requests has increased over time. Driving the demand are new treatments for serious unmet medical needs; patient advocacy groups pressing for access to emerging treatments; internet platforms enabling broad awareness of compelling cases or novel drugs and a lack of trust among some that the pharmaceutical industry and/or the FDA have patients' best interests in mind. High-profile cases in the media have highlighted the gap between patient expectations for compassionate use and company utilisation of fair processes to adjudicate requests. With many pharmaceutical manufacturers, patient groups, healthcare providers and policy analysts unhappy with the inequities of the status quo, fairer and more ethical management of compassionate use requests was needed. This paper reports on a novel collaboration between a pharmaceutical company and an academic medical ethics department that led to the formation of the Compassionate Use Advisory Committee (CompAC). Comprising medical experts, bioethicists and patient representatives, CompAC established an ethical framework for the allocation of a scarce investigational oncology agent to single patients requesting non-trial access. This is the first account of how the committee was formed and how it built an ethical framework and put it into practice.

\section{INTRODUCTION}

In the USA, the public policy goal underlying the regulatory approval of drugs is ensuring that only safe and effective drugs reach the market. This requires extensive animal and human testing. But individuals who are dying or seriously ill often perceive themselves, rightly or wrongly, as unable to wait for these new drugs to be approved. They seek to maximise their individual chances of cure or remediation, while the regulatory process is oriented towards medical products for use by populations. In this context, there is a fundamental moral tension between protecting the public and responding to the plight of very ill individuals. The latter may require nothing more than allowing informed, consenting individuals to pursue any chance regardless of lack of information about efficacy or safety. But, creating a system in which informed, consenting individuals can try anything would destroy the clinical trials that prove a new product's worth, because those individuals would have no reason to risk being randomised to a placebo or standard of care arm in a trial. The unintentional consequence would be harming the public that the regulatory system is supposed to protect. It is clear that neither a framework that values only individual ends nor one that values solely public ends is desirable.

Patients who seek individual access to experimental treatments, sometimes called 'compassionate use' or 'expanded access,' have exhausted all available options. They are unable to benefit from existing, approved products; they do not qualify for clinical trials for a variety of reasons; they cannot enrol in an expanded access programme making the experimental treatment during the period between a clinical trial's end and regulatory approval of the product or no such programme exists. For such patients, the only option is to seek access to an experimental treatment from a pharmaceutical company. Pharmaceutical companies, however, are not responsible for trying to save individual patients. Their mission is to develop products that will ultimately gain approval from the FDA and other regulatory bodies so they can sell them to all patients in need.

In the case of many drugs in development, the supply of product available for compassionate use requests is inadequate to meet patient demand. Companies often create only limited quantities of experimental products; they can be difficult to produce due to a complex supply planning process that includes uncertainty of approval, uncertain demand and limited shelf life. If a company is willing to grant requests for compassionate use, it must decide who among those requesting the product will receive it.

In some cases, companies make decisions about granting compassionate use solely on medical grounds. In other cases, some method of allocation or rationing, such as first-come, first-serve, has been used. With no widely agreed-on best practices, and 
with such decisions made out of the public's view, allocation may be criticised as unfair. For example, companies that grant access to someone who is famous, wealthy, politically connected, socially or physically appealing (a young child, a newlywed) or who has access to certain influential experts may do so at the expense of those who lack those attributes. Basing allocation decisions for a potentially scarce resource that, in the best case scenario, may be life-saving on such factors or qualifications is difficult to defend, and reports of such incidents incite anger among those who see them as evidence of unfairness in responding to compassionate use requests. In this paper, we report on a pilot programme developed to make as fair as possible compassionate use allocation decisions for a promising experimental drug that was in very short supply. This pilot, called the Compassionate Use Advisory Committee (CompAC), was a collaboration between the New York University (NYU) School of Medicine Division of Medical Ethics and Janssen Pharmaceuticals, a Johnson \& Johnson company. This is the first detailed account of how the committee was formed and how the ethical framework it developed was conceived and put into practice.

CompAC was a diverse, international panel of experts in medicine, bioethics and patient advocacy that provided Janssen with recommendations about whether to allocate an investigational drug in response to individual compassionate use requests. At the outset, this committee collectively discussed various methods by which to allocate a scare resource when medical criteria alone were insufficient, including first-come, first-served; lottery or a set of guiding principles. Working closely with the company and external experts in the disease, clear medical criteria were defined for all patients being considered based on available data from the drug's clinical development programme. Importantly, it was unanimously decided that a set of principles would be the preferred method for a situation in which the resource was likely to benefit some, but not all. It was also decided that Janssen medical and clinical teams must keep CompAC apprised of new data about the drug's safety and efficacy, so that CompAC could, as necessary, recalibrate the predefined criteria and its recommendations.

\section{THE CRITICAL NEED FOR A FAIR PROCESS}

Patients have sought and received experimental pharmaceuticals outside of clinical trials for decades, yet disagreement about the wisdom of using experimental agents continues and pharmaceutical manufacturers continue to encounter logistical, financial, ethical and other problems with the practice. ${ }^{1}$ There are no industry-wide best practices, and many companies that have granted compassionate use, or preapproval, access to their products have done so quietly, without fanfare and without divulging the process or grounds on which decisions were made. No systematic studies have been published concerning these processes.

The number of compassionate use requests has increased over time. ${ }^{2}$ Driving the demand are new treatments for high life-threatening, unmet medical needs, some of which are for conditions that have not previously had therapies; patient advocacy groups pressing for access to these treatments as early as possible; social media platforms enabling broad awareness of compelling cases for drugs in development and a lack of trust among some that the pharmaceutical industry and the FDA have patients' best interests in mind. The media's interest in dramatic stories, such as those of dying patients' quests against long odds for successful treatment via experimental drugs, also fuels interest. Recent high-profile cases highlight the gap between the expectations of individual patients seeking drugs through compassionate use and the general availability of organised and fair processes to adjudicate such requests. ${ }^{3}$ With many pharmaceutical manufacturers, patient groups, healthcare providers and policy analysts unhappy with the inequities of the status quo, fairer and more ethical management of compassionate use requests was needed.

\section{THE PILOT PROJECT}

Janssen Pharmaceuticals, operating in this context while managing compassionate use requests for its agents, looked for guidance from an outside organisation. It asked the NYU School of Medicine Division of Medical Ethics to develop a new model for handling compassionate use requests in a fair, transparent and justifiable manner. The Janssen/NYU collaboration agreed to form an independent entity, managed by the Division of Medical Ethics, to function in an advisory capacity. This entity was named the Compassionate Use Advisory

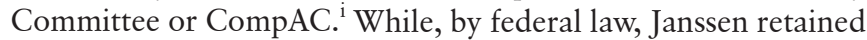
decision-making authority over distribution of its unapproved drugs, the company committed to be guided by the independent CompAC's recommendations.

For the pilot, Janssen requested allocation guidance for an oncology agent, daratumumab, being developed as a treatment for refractory multiple myeloma, a bone marrow cancer that kills more than 12000 people annually in the USA. ${ }^{4}$ At the time of the pilot's launch, daratumumab was in extremely short supply for use outside of early clinical trials as it was not approved by any health authority and faced demand in larger-cohort expanded access programmes. A biologic, the agent is complicated and time consuming to make. Although Janssen had produced enough to conduct clinical trials, positive early results indicating efficacy and a tolerable safety profile further exacerbated supply concerns. Janssen attempted to ramp up production in the increasingly likely event that health regulators would grant early approval of the drug. Thus, CompAC was initially faced with deciding who should get an investigational drug and who should get it if there were not enough for all eligible patients. Insufficient supply was rare, as Janssen had worked to minimise scarcity by modifying its supply chain and having its clinical teams reroute the drug, in some cases hand-labelling it for the compassionate use programme. At the same time, it did not want to risk a shortage that would disrupt the larger clinical development programme or impair its ability to provide the drug commercially after regulatory approval.

The Janssen medical team determined which requests would be sent to CompAC based on predefined criteria, and the company agreed to cover the costs of providing the drug. Requests from patients (via their physicians) who had not yet tried all approved and available multiple myeloma treatments were met with instructions to try them. Requests from patients eligible to enrol in clinical trials for the disease were met with information about suitable trials, and requests from patients considered to have unacceptable safety risks were denied. Requests from patients who had tried all approved medicines and were eligible to receive the drug but unable to enrol in clinical trials or any of Janssen's other programmes to access daratumumab were referred to CompAC. ${ }^{\text {ii }}$

${ }^{\mathrm{i} D e t a i l e d ~ i n f o r m a t i o n ~ a b o u t ~ t h e ~ c o l l a b o r a t i v e ~ e f f o r t ~ a n d ~ t h e ~ q u e s t i o n s ~}$ it dealt with in creating CompAC will be discussed in a separate paper.

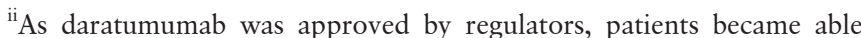
to access the drug through normal channels. In some countries, as the drug was going through the approval process, Janssen created expanded 
Six months after the launch of the CompAC pilot, daratumumab (Darzalex) was approved for use in the USA, ${ }^{5}$ and American physicians began to prescribe it immediately. The daratumumab shortage for individual compassionate use requests was relieved soon after approval, on 21 November 2015, as several other large trials were delayed or cancelled, freeing more drug for compassionate use. The drug has since been approved in many other countries and remains under review in a few others. CompAC continued to receive individual compassionate use requests from other parts of the world through early December 2016. CompAC is still available, if necessary, to review requests for access for patients who cannot otherwise access the drug and who live in areas where the drug is unapproved.

\section{WHO SHALL ADVISE? CREATING COMPAC}

Compassionate use requests for daratumumab from patients in countries in which the drug was intended to become commercially available eventually were submitted, after review and screening by Janssen, to the independent CompAC. The committee comprised 10 men and women from the USA, UK, Canada, Poland and Lebanon and included a non-voting chair and deputy chair from the Division of Medical Ethics. The national/geographic diversity of the group was intentional, as requests would be coming from around the world. As a collective, members offered expertise in investigational drug use in cancer and other diseases, bioethics and patient advocacy perspectives not connected to multiple myeloma. This varied expertise was seen as critical to CompAC's credibility with doctors, patients and families. CompAC also had access to independent multiple myeloma experts who had been contracted to be available as needed by the committee.

CompAC members had to be willing and able to make allocation decisions that patients and their physicians considered to be matters of life or death. Committee members understood that the supply of daratumumab was limited and therefore that some requests may have to be denied. Given this, approximately one of every four people approached about serving on CompAC declined. And yet, when the creation of CompAC was announced in the media, the Division of Medical Ethics heard from a flood of volunteers wishing to serve on the novel committee. ${ }^{\text {iii }}$

Before CompAC began reviewing requests, it went through an extensive orientation, in which the members learnt about daratumumab and multiple myeloma; reviewed literature on allocation decisions and the methods by which they have been made; practiced reviewing mock cases; and developed bylaws and guidelines for themselves.

\section{PROCEDURAL FAIRNESS, BLINDING AND SPEED THRILLS}

The CompAC pilot was officially launched on 7 May $2015 .^{6}$ Some patients who seek access to investigational drugs outside

\footnotetext{
access programmes (EAPs), programmes that are meant to provide experimental drugs to groups of patients without them having to request it individually. In countries where EAPs were created, the Janssen medical review team checked to see if a patient was eligible to receive drug via an EAP; if so, the patient was referred to the EAP instead of CompAC. When, in several situations, the number of patients requesting the drug exceeded the available slots in the EAPs, those patients who were not accommodated by the EAP were referred to CompAC. CompAC made a decision that all of these patients, having been considered eligible to receive the drug via EAP, would receive a recommendation from CompAC to be granted access to daratumumab.

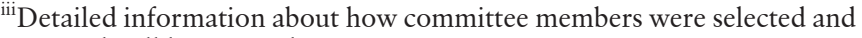
recruited will be covered in a separate paper.
}

of clinical trials complain about a slow, or no, response from companies. A key dimension of fairness in the CompAC process was the commitment to respect patients by acknowledging and reviewing their requests rapidly. CompAC's bylaws specified that requests would be heard on an emergency basis within 24 hours if necessary by the chair and deputy chair; otherwise, weekly meetings were scheduled so that every case referred to the committee would result in a recommendation to Janssen within five business days.

Weekly meetings of the chair, deputy chair, staff and as many committee members as could be available (always more than quorum) were held, via teleconference and the internet, to consider requests. Prior to each meeting, members reviewed the requests and learnt how much drug was available for allocation. Requests were submitted via a template developed by CompAC and Janssen to ensure that uniform information would be available about each patient. In order to neutralise the influence of bias, CompAC members decided to blind themselves to some information. Names of patients, doctors and countries of origin were redacted, as were patients' race, gender and ethnicity. Blinding, along with CompAC's independence from Janssen, helped to establish the fairness that was essential for the committee to gain the trust of physicians and patients. The committee did ask that patients' ages and medical histories be included as well as whether a patient was solely responsible for dependents (defined broadly).

For each meeting, three CompAC members-representing the clinical, bioethical and patient perspectives-were selected from the pool of deliberating members to vote on whether to recommend that Janssen allocate daratumumab. Voters' identities were withheld until a day before each meeting, to preclude lobbying by or other pressure from interested parties. Meetings consisted of an 'open session,' during which the chair, deputy chair, CompAC members, staff, external multiple myeloma experts and at least one member of Janssen's daratumumab medical affairs team participated in reviewing and discussing cases. The chairs and staff then oversaw a 'closed session', during which that week's three voting members deliberated and voted on three possible options: approve the request, decline the request or ask Janssen for more information about the patient. ${ }^{\text {iv }}$ The committee always received the information it asked for, and Janssen consistently followed CompAC's recommendations. Cases that CompAC declined were occasionally returned to the committee if relevant clarifications or new information became available, such as updated lab results. The numbers of cases and recommendations through 31 December 2015 have been reported elsewhere. ${ }^{7}$

Fairness was achieved by creating a rapid response to all requests, ensuring independence from the sponsor, creating a single route of entry into CompAC and a standard form to be used for all cases, and by 'double-blinding' the requests to the committee and the identity of voting members in any given week. In addition, CompAC pressed Janssen to see that the company's international websites were easy to navigate for those seeking information about compassionate access to daratumumab (consistent with local and regional guidelines restricting

\footnotetext{
${ }^{\text {iv }}$ At the start of the pilot, only two options were available: yes or no. This was revised after the need for a third option was identified. Before the third option was added, CompAC twice voted to decline a recommendation but informed Janssen that if specific information were made available to the committee, it would recommend that drug be provided. In both cases, Janssen procured the additional information and, following the guidance from CompAC, allocated the drug. Thus, in two instances Janssen did not follow the letter of the CompAC recommendation but did follow the spirit of it.
} 
off-label promotion). Without strenuous efforts to promote fairness, CompAC would have been doomed to fail, no matter the criteria on which it based its actual decisions.

\section{ESTABLISH, REVIEW, ADJUST...REPEAT}

Before CompAC began to review requests, the members, chairs and staff debated how they might evaluate individual cases. They considered a random lottery process and a first-come, firstserved protocol, but these were rejected as inappropriate for the allocation of a likely to be effective oncology agent. A lottery would fail to capture nuances in patients' health status and thus their suitability for the drug, and a first-come, first-served system could unfairly favour those with physicians better informed about drugs in development and patients with easier access to top quality healthcare and facilities. ${ }^{8}$ CompAC members felt that potentially life or death decisions ought to be made on cases' individual medical characteristics and likelihood of benefit to the patient and decided to establish a set of principles and relative weightings for them as a basis for decisions. This was not intended to be a standardisable document; rather, it was specific to patients with multiple refractory multiple myeloma, a disease that tends to appear in older populations. ${ }^{4}$ The principles the committee arrived at were devised to establish a just and fair process for all patients, to incorporate scientific and medical evidence into decisions and to take into account patient perspectives and needs.

Operating under a presumption of scarcity and considering the welfare of potentially very sick patients, CompAC put a higher allocation priority on cases in which, first, no known or unacceptable harms were anticipated (nonmaleficence); second, whether there was scientific/medical evidence supporting the hypothesis that daratumumab could benefit the patient (efficacy and beneficence) and, third, whether the patient was fit and stable enough to tolerate the therapy and to realise clinical improvements for a reasonably sustained period. Each of these involved judgment and best guesses. Clinical trials were ongoing, so it was possible that accumulating evidence would indicate, for example, that the investigational drug had some sort of toxicity or worked best in a specific type of patient. To make the best and most scientifically informed decisions possible about both potential risks and benefits to the patients, CompAC was briefed extensively about multiple myeloma and the drug before beginning its work and updated at regular intervals with ongoing trial results. It also had its own independent multiple myeloma experts and the Janssen daratumumab medical and clinical teams as resources when specific questions about safety or efficacy data arose. However, no matter how closely the trial data were studied, the fact remained that the patients whom CompAC encountered were unable to participate in clinical trials; thus, trial data could not be considered to be absolutely predictive.

The issue of patient fitness was central to the committee's deliberations, but it also bedevilled the project. Multiple myeloma is a cancer of the bone marrow, and it has numerous symptoms that affect patients' ability to receive systemic therapy, including low red or white blood cell or platelet counts, impaired kidney and lung function and diminished overall performance status or ability to care for themselves. Patients may become anaemic; they may develop bone lesions that can escalate to bone fractures; they may need dialysis or they may have significant pain and disability. ${ }^{9}$ In considering fitness, the committee sought to prioritise patients whose physical state could possibly be helped by the drug. For example, daratumumab might be able to prevent the development of new bone lesions, but it cannot repair existing ones. There was a chance that it might have positive impact on both renal dysfunction and anaemia; however, there was little hope that treatment with daratumumab would restore a bedbound patient to activity.

CompAC sought to distinguish those patients whom daratumumab might be able to, at least temporarily, restore to functionality or relieve of significant complaints from those who, even if daratumumab were beneficial, would still experience serious symptoms or limitations of function from their disease. Although there was consensus among CompAC members that such distinction between patients was meaningful, no good tool for differentiating between the classes of patients was identified. Ultimately, and with recognition of its shortcomings as a proxy for the sort of distinctions CompAC sought to use, the group evaluated each patient by looking at a combination of functional status measurement (eg, Eastern Cooperative Oncology Group (ECOG) score ${ }^{10}$ ), comorbidities, metabolic profiles and physician narratives. Physicians were also asked to provide independent activity of daily living values for patients, although the vast majority of treating physicians did not do this and provided ECOG performance status scores instead.

While possibility of benefit and avoidance of harm were the dominant principles that guided CompAC, several other factors were given subordinate ('tie-breaking') status. One of these was age. After discussions with its independent experts, CompAC did not believe age to be an important factor for evaluating requests, but it agreed to consider a significant difference in patient age when (1) there were more requests than drug available and (2) there was no significant difference between otherwise similarly situated patients. For example, if there was only enough daratumumab to allocate to one patient but two patients were felt to be equally suitable candidates, a significant age difference would tip the scale towards the younger patient. This 'significant age distinction' would come into play in the above case if the two patients were, say, 43 and 85 , but not 54 and 56. In general, because fitness was considered to be a better indication of suitability than age, a fit older patient would have been prioritised over a less fit younger patient. These prioritisations were rarely applied.

Along with age, there were other lower-order principles that CompAC used only when drug supply was insufficient to accommodate all the requests the committee deemed appropriate. One of these was whether a patient had dependents. This question was worded specifically: 'Is the patient solely responsible for dependents (for example, spouse or partner, child(ren), siblings, other relatives, or any other individual dependent on the patient for financial and/or personal care support)? If so, please provide details'. The committee was interested in whether others would suffer financial or physical hardship were the patient to die; while all friends and family would suffer from the loss of a loved one, CompAC deemed the loss of a sole provider (be that of money, lodging, caregiving or such) to be worthy of some consideration. Given the little that was known at the time about how long daratumumab might continue to work in patients, it was considered reasonable to view this as a possible long-term therapy during which patients would be able to work, live independently and perform caretaking activities. Importantly, CompAC did not ask for any other social information (such as marital or parental status, occupation, income and the like) from patients.

These principles and their relative weightings could serve only as macrolevel guides for individual cases; they could not produce anything close to automatic decisions. Thus, deliberation on the independent merits of each case was warranted. The initial shortage of daratumumab and the similarity among many 
of the patients impelled the committee to look for microlevel differences among patients. In practical terms, this meant that CompAC needed to make distinctions for allocation decisions that would not always be relevant to clinical decisions. For example, measures of renal or respiratory function could vary significantly among patients and still not affect clinical decisions to use daratumumab, while the same degree of variation in those measures could favour some patients over others when daratumumab was in extreme shortage in a particular week. Certain differences in functional status measures may not be important to clinical decisions to use the drug, but they can be to allocation decisions in the context of scarcity. And, as noted, the secondary considerations of age and dependents came into play only in situations in which patients were similar with regard to their perceived risk and benefit but scarcity of drug demanded choices to be made.

Of note, in weeks of extreme scarcity of drug, even these microlevel decisions were not always sufficient to guide the committee's recommendations. There were occasions when CompAC deliberated whether, according to its principles, it should deny the request of a less than ideal recipient and instead store ('bank') an available dose of the drug in the event a 'more promising' candidate came to the committee the following week. Although reservations were expressed, this policy was followed early on, during the period of the greatest limit on supply. As supply loosened, CompAC did not have to consider whether to bank doses for future patients.

CompAC initially tested its principles and procedures on actual cases; the committee did not render recommendations, leaving the decisions to Janssen as had hitherto been done. During that phase, patient age became a more important factor in deliberations than anticipated because of a higher than expected number of cases involving comparatively young patients.

At various intervals during the pilot, CompAC reviewed its previous cases and revisited its principles to see whether corrections needed to be made. The committee had requested information about whether patients had participated in a clinical trial. It felt trial participation, which generates data for scientific advancement and the benefit of others, to be worthy of recognition, viewing it as akin to a 'bonus point' that could differentiate among patients, in weeks when supply would not meet demand, who had otherwise been deemed equal. Patients who could not participate in trials (eg, due to comorbidities) were not penalised.

Later in the pilot, another consideration was added into the mix of secondary considerations. This was deference to those patients who had endured a significant burden of illness over time. Although every case that came before CompAC described patients who had tried all available approved therapies for multiple myeloma and whose cancer continued to progress, some of the patients' histories showed stable periods when a therapy had worked or the disease had otherwise been in check. Other patients had either no such periods or none of a significant length of time. In the rare case where these relatively long-suffering patients seemed appropriate to receive daratumumab, CompAC considered their significant burden of illness as a secondary indication sufficient to differentiate them from others presenting very similar profiles.

\section{FACTORS COMPAC DID NOT TAKE INTO CONSIDERATION IN MAKING RECOMMENDATIONS}

CompAC did not ask whether patients were married, employed, parents, privately or publicly insured, had a criminal history or any social questions aside from whether the patient was the sole support for some sort of dependent. The committee did not consider these to be morally appropriate or germane, and some members recalled the 'God Committee', a group empaneled to help a Seattle hospital decide who among eligible patients would receive access to a highly limited, expensive new treatment known as dialysis. Shana Alexander's November 1962 article $^{11}$ on the group sparked public outrage that life or death treatment decisions had been based, in some cases, on social criteria. CompAC members were united against considering such factors in their decisions, and the standardised form that physicians completed to request daratumumab for a patient provided no space for such information. The deputy chair reviewed all requests before they were sent to CompAC members, and social information, as well as other information CompAC decided should be blinded, was redacted.

One consideration that CompAC wanted to use in its deliberations was what patients themselves wanted. Thus, an area marked 'patient narrative', where patients would be able to speak directly to the committee, was included on the form. The committee received no narratives; more often than not the requesting physicians just copied the patient's medical history into this space, and CompAC was unable to factor in patients' expressed wishes. One reason may be that the form was not clear about what it was asking. Another is that the physicians may have been filling out the form without the patient present to provide explicit preferences.

\section{FOR FURTHER ETHICAL CONSIDERATION}

During the course of the pilot, many ethical issues arose that had not been forecast at the outset. One was that of drug dose: daratumumab dosing is based on weight, and the amount of drug needed can vary significantly from one patient to another. In a situation of extreme scarcity, should one heavier patient receive a quantity of daratumumab sufficient to treat two smaller patients? This question remained hypothetical, as CompAC never had to face this circumstance.

Another issue was what trying 'all approved options' really meant. This first arose when CompAC and its independent medical experts wondered why some patients had not had bone marrow transplants when others had had several. After discussion, it was decided that 'all available treatment options' need not include bone marrow transplants, because guidelines about patient suitability for the procedure vary around the world, as does access to a number of other therapies for the treatment of myeloma.

Individual compassionate use programmes are meant to be the last resort for patients. 'Last resort' generally refers to the absence of any alternative treatment options, either because alternatives do not exist or they cannot be used for clinical reasons. But treatment options can also be out of reach to patients for other reasons: a treatment generally available in many regions is not approved for use in the country in which the patient resides; clinical trials and expanded access programmes involving the experimental treatment are too far away for the patient to participate and treatments considered appropriate by treating physicians are not covered by private health insurance or government programmes. Individual compassionate use programmes could be seen as a way to circumvent or address any of these factors and in effect broaden their scope beyond the original design. Patients who request compassionate access because they cannot pay for clinically available options could lead payers (public and private) to direct patients to these 
programmes to avoid covering treatments. Less clear are situations in which clinical trials or expanded access programmes are available but patients claim that they cannot participate because of practical reasons or convenience. Thus, the challenge for CompAC and other organisations making compassionate use decisions is to determine what constitutes last resort scenarios beyond just whether clinical alternatives exist or not, and how to accommodate these scenarios in any decision process. These and other such issues underscored that CompAC in no way was able to create an algorithm to decide responses to compassionate use requests.

Some of the requests tempted CompAC members to question a patient's medical treatment. A compelling argument can be made for not giving an unapproved investigational drug to patients if there is a likelihood that tweaking their current, approved, medications could benefit them. However, second-guessing treatment regimens would move CompAC into the healthcare professional's sphere of authority, so committee members made conscious efforts to distinguish between concerns relevant to allocation recommendations and those about how a case was being managed in general. The latter were relayed to Janssen to pass to the treating physician.

Tracking patient race and ethnicity was a contentious issue for CompAC. Multiple myeloma outcomes vary by race, ${ }^{4}$ and while there are undoubtedly many reasons, both biological and social, for this, there is evidence that certain phenotypes are associated with worse outcomes. CompAC was divided on whether it should request racial and/or ethic information about patients, and it was pragmatically concerned about what sort of answers such a question may provoke, given that requests were coming from around the world and not every nation uses the same racial or ethnic constructs as the USA. This issue was partially resolved after Janssen informed members that by law it was barred from asking for this information in certain nations. To keep the request form standardised across the globe, the committee asked for no information about patient race or ethnicity.

Another recurring topic of discussion was the fact that physicians who received access to daratumumab for their patients were not required to report to Janssen information beyond adverse events felt to be linked to the drug. Indeed, in some nations, this was all the information Janssen was permitted to require of the treating physician. However, CompAC repeatedly sought information about patient outcomes. Did patients who received daratumumab via compassionate use benefit? Were they harmed? And if some benefited and others did not, were there commonalities that might be useful in predicting which future requesters would be more likely to benefit? For those who benefited, was their quality of life sufficiently tolerable to offset any side effects of the drug? For the pilot, Janssen was unable to supply CompAC with outcome data on patients whom the committee had recommended for treatment; it provided only the number of resupply requests for daratumumab it received, which is a proxy for survival. This situation represents a lost opportunity to generate real world evidence for an unapproved drug not otherwise available to clinicians, regulators and payers as well as to support CompAC's effort to make decisions based on maximising the chance of benefit.

Finally, a finding of concern to CompAC is that postvote unblinding of data (which was done occasionally to check for any signs of bias in the committee's decisions) revealed that the majority of the requests had come from a relatively small number of physicians; also, the number of requests per eligible country varied dramatically. If these repeat requestors constituted the entirety of oncologists who treat patients with multiple refractory multiple myeloma, this would not matter. However, the committee suspected that requests came from only a small number of those who treat such patients. Why were some physicians requesting compassionate use access to daratumumab for their eligible patients while others were not? Was it because not all physicians knew about the availability of the drug via compassionate use, the result of local restrictions on promotion and distribution of unapproved drugs or because of variations in their training? Or was it because some were either unable or unwilling to navigate the request process, which was in English? Was it because only certain patients were interested in compassionate use and able to surmount the hurdles it required, such as that it must given through repeated lengthy infusions in a qualified medical centre? This was a situation that would be impossible for CompAC to evaluate or even influence, yet it had very real consequences for the goal of designing a fair system of access at the national and global health levels.

\section{WHAT HAS BEEN LEARNT AND WHAT NEEDS TO BE LEARNT?}

The CompAC pilot experience showed that a medical ethics division within a large university could assemble a group of people from around the world with expertise in oncology and medicine, bioethics and patient advocacy to make fast, medically based allocation recommendations for an individual compassionate use programme while grappling with uncertainty in light of incoming information about the experimental drug and under conditions of severe scarcity. Members were able to agree to general allocation principles and relative weightings for various circumstances. The pilot has also shown that it is possible to establish and integrate IT support and logistical operations necessary to receive patient requests in standardised, redacted, secure, blinded formats, conduct case decision meetings on a weekly basis with members in disparate global locations, adjudicate and record decisions and return recommendations to a sponsor in an expedited manner. Weekly meetings were cancelled only when no requests had been received or there was no drug to allocate; no meetings were cancelled because of member attendance problems, operational failures or discord among members.

The diversity in expertise among CompAC members proved to be as important to deliberations as anticipated. The clinical management perspective can discern patients more likely than others to benefit or experience harm from a treatment. Because this perspective tends to focus on compelling reasons why a particular patient may not be a good candidate for an experimental treatment, it would not always differentiate patients to the degree needed in extreme scarcities. In contrast, an allocation perspective tends to focus on compelling reasons why a particular patient may be a good candidate and can further differentiate patients who represent good candidates on clinical grounds. But an allocation perspective can also identify good candidates based on ethical and other principles that do not account for important clinical dimensions. Therefore, the committee evidenced the necessary tension between the clinical and allocation perspectives. The patient advocate perspective kept the clinical and allocation debates centred on patient welfare. The principles and processes applied produced recommendations acceptable to Janssen.

\section{CONCLUSION}

The early experience of CompAC suggests that an academic centre can assemble a group of people who bring an expertise in medicine, bioethics and patient advocacy and develop a fair, 
transparent and useful individual compassionate use programme involving an experimental drug in short supply. The composition of the committee also shows that its disparate members can reach agreement on guiding principles, draw on these principles when making fair allocation decisions for individual cases and learn from their experience as the choices they faced evolved over time. The arrangement can take the form of collaboration with the manufacturer of the experimental treatment, in which the manufacturer retains the authority on individual cases but is at arm's length from the independent deliberation that results in recommendations.

The collaboration between the NYU School of Medicine Division of Medical Ethics and Janssen Pharmaceuticals that led to the CompAC pilot is the first response to the recent debates about the best way for pharmaceutical manufacturers to manage individual compassionate use decisions. The arrangement would appear to be a promising response to the need for a fair and accountable process when the treatment sought is in very short supply. Other approaches may be better for other treatments and sponsors, or may be better altogether, and so others should be considered and piloted. Furthermore, the principles and ordering selected by CompAC were in response to a particular drug for a particular disease and cannot be applied across the board to other contexts, although the overarching ethical principles of nonmaleficence, beneficence and justice can and should be applied universally.

The need for fair and just processes for individual compassionate use decisions will only intensify. Advances in molecular biology and genetics are identifying new mechanisms that cause diseases and creating new treatments directed at them. Patients and physicians are demanding access to promising treatments earlier and earlier, and they are turning more to social and mainstream media to make their cases. Efforts to find the best ways to expand fair access to experimental treatments for people who have no other options, therefore, must continue.

\section{Author affiliations}

'Division of Medical Ethics, NYU School of Medicine, New York City, New York, USA

${ }^{2}$ Brookfield, Connecticut, USA

${ }^{3}$ Department of Medical Oncology, Sidney Kimmel Cancer Center, Jefferson

University, Philadelphia, Pennsylvania, USA

${ }^{4}$ Salim El-Hoss Bioethics \& Professionalism Program, Faculty of Medicine, American University of Beirut \& Medical Center, Beirut, Lebanon

${ }^{5}$ Dalla Lana Faculty of Public Health, University of Toronto, Toronto, Ontario, Canada ${ }^{6}$ Department of Family and Community Medicine, Sinai Health System, Toronto, Ontario, Canada
${ }^{7}$ Department of Psychiatry, Wellcome Centre for Ethics and Humanities, University of Oxford, Oxford, UK

${ }^{8}$ Center for Bioethics \& Biolaw, Institute of Philosophy, University of Warsaw, Warsaw, Poland

${ }^{9}$ Muscular Dystrophy Association, Tucson, Arizona, USA

${ }^{10}$ Department of Pediatrics, NYU Langone Health, New York City, New York, USA

Acknowledgements The authors and committee are grateful to John Walsh, Edward Stadtmauer, Shaji Kumar, and Ola Landgren for their contributions to CompAC discussions and deliberation. The authors and committee thank Jessica Wico and Beba Blagojevic for their ongoing organisational assistance with CompAC.

Collaborators Members of the Compassionate Use Advisory Committee: $\mathrm{E}$ Mitchell, T Arawi, R Upshur, I Singh, J Rozynska, V Cwik, S L Gardner.

Contributors ALC, JRT, LK and ASBH all contributed to drafting, writing and editing of the manuscript. Other CompAC members provided feedback.

Funding The authors have not declared a specific grant for this research from any funding agency in the public, commercial or not-for-profit sectors.

Competing interests Janssen Pharmaceuticals provided administrative costs. First author and NYU-affiliated authors are not paid. Some CompAC members take payment, others do not.

Patient consent Not required.

Provenance and peer review Commissioned; externally peer reviewed.

\section{REFERENCES}

1 Darrow JJ, Sarpatwari A, Avorn J, et al. Practical, legal, and ethical issues in expanded access to investigational drugs. N Engl J Med 2015;372:279-86.

2 Jarow JP, Lemery S, Bugin K, et al. Expanded access of investigational drugs: the experience of the center of drug evaluation and research over a 10-year period. Ther Innov Regulatory Sci 2016;50:705-9.

3 Caplan A, Moch K. Rescue me: the challenge of compassionate use in the social media era. Health Affairs Blog. Project Hope: The People-to-People Health Foundation, Inc. (cited 2018 Jan 12).

4 cancer.org. Atlanta: American Cancer Society; c2018. cited 2018 Jan 12. https:// cancerstatisticscenter.cancer.org/?_ga =2.128003656.538881780.15157675131926856078.1515767513\#!/cancer-site/Myeloma

5 Daratumumab (Darzalex). fda.gov. Silver Spring (MD): U.S. Food \& Drug Administration. https://www.fda.gov/Drugs/InformationOnDrugs/ApprovedDrugs/ ucm530249.htm (cited 2018 Jan 12).

6 Thomas K. Company creates panel for access to trial drugs. The New York Times, 2015. Sect. A:1.

7 Caplan AL, Ray A. The ethical challenges of compassionate use. JAMA 2016:315:979-80.

8 Persad G, Wertheimer A, Emanuel EJ. Principles for allocation of scarce medical interventions. Lancet 2009:373:423-31.

9 cancer.net. Alexandria (VA): American Society of Clinical Oncology (ASCO); c20052018. cited 2018 Jan 12. https://www.cancer.net/cancer-types/multiple-myeloma/ stages.

10 ecog-acrin.org. Philadelphia: ECOG-ACRIN Cancer Research Group; c2017. cited 2018 Jan 12. http://ecog-acrin.org/resources/ecog-performance-status

11 Alexander $\mathrm{S}$. They decide who lives, who dies: medical miracle and a moral burden of a small committee. Life. 1962. 